\title{
MAXIMIZATION OF FLIGHT TIME FOR AIRPLANES WITH ELECTRICAL DRIVE BY POWERPLANT OPTIMIZATION
}

\author{
S. Serokhvostov \\ Central Aerohydrodynamic Institute (TsAGI), Zhukovsky St., 1, Zhukovsky, 140180, Moscow Region, Russia \\ Moscow Institute of Physics and Technology (MIPT), Department of Aeromechanics and Flight Engineering (DAFE), \\ Gagarina St.16, Zhukovsky, 140180, Moscow Region, Russia.E-mail: serokhvostov@aviel.ru \\ Received 1511 2006, accepted 11062007
}

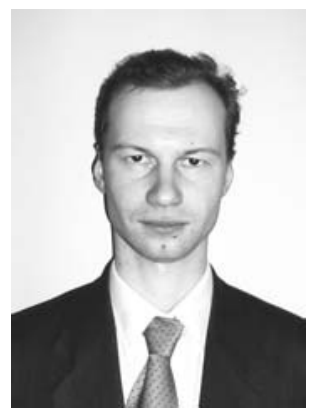

Sergey SEROKHVOSTOV, Dr Sc Eng

Date and place of birth: 1975, Moscow Region, Russia.

Education: Moscow Institute of Physics and Technology (MIPT) 1998, PhD 2002.

Present position: Assoc. Prof. (Chair of "General Physics" MIPT DAFE), Vice Chair of "Flight Engineering"

(MIPT DAFE), Researcher (TsAGI)

Publications: more than 10 scientific and educational papers

\begin{abstract}
In this paper, the problem of maximizing the flight time of an airplane with an electrical power plant (AEP) by the optimization of the mass of the accumulator in cases of fixed and non-fixed airframe is considered. Variants of high (turbulent flow) and low (laminar flow) Reynolds numbers are taken into account. Dependence of flight time on airplane parameters is obtained. The behaviour of flight time as a function of accumulator mass near the maximum is also investigated. A comparison between the results obtained and the data for the existing AEP is made. On the basis of the results obtained, the influence of aircraft parameters on flight time is analyzed.
\end{abstract}

Keywords: airplane with electrical power plant, accumulator, optimization, flight time

\section{Introduction}

One of the main characteristics of airplanes with an electrical power plant (AEP) is flight time. One of the peculiarities of such a power plant is constant mass during the flight (compared to power plants with piston engines and jet engines).

Two ways of increasing flight time exist: by optimal control during the flight and by improving the construction of the aircraft. The best result occurs when we use both these methods simultaneously.

It was shown that the optimal control algorithm for maximizing flight time must be as follows: the airplane must accelerate to cruise velocity using the maximum power of the power plant and then continue the flight at cruise velocity till the onboard energy is completely consumed [3]. Cruise velocity corresponds to the state of minimal energy consumption.

The conventional EP consists of the electrical drive, accumulator, and control devices. It can be assumed that the mass of the accumulator is proportional to its energy and the mass of the drive is proportional to its power.

On the one hand, an increase in the mass of the accumulator increases onboard energy and can increase flight time. On the other hand, an increase in mass increases power consumption (and maybe the mass of the electrical drive and control devices) and decreases the flight time. So, there must be an optimal mass of the accumulator for maximum flight time.

Considered are problems of flight time maximization through the optimization of power plant mass for the following tasks:

1. Optimization of the accumulator mass for the fixed airframe for cruise flight. This task corresponds to the problem of improving the characteristics of the existing aircraft.

2. Optimization of the power plant mass in the case of non-fixed airframe parameters for cruise flight. This task corresponds to the preliminary design optimization problem. In this problem, it is assumed that the masses of some airframe elements depend on the total mass of the aircraft, and the mass of the electric drive depends on the power consumed.

These problems are investigated for two cases:

1. Constant value of the drag coefficient at zero lift $C_{\mathrm{D} 0}$ (high values of Reynolds number $\mathrm{Re}$ ),

2. $\mathrm{C}_{\mathrm{D} 0} \sim \operatorname{Re}^{-1 / 2}$ (low $\mathrm{Re}$ ). 


\section{Optimization of the accumulator mass for the fixed airframe for cruise flight at low Re}

In this section, we assume that we can change only the mass of the accumulator (i.e., the energy of the accumulator), and cannot change other components of the airplane. This corresponds to the problem of improving the existing MAV by the correct selection of the mass of the accumulator.

Also assume that the efficiency of the power plant at cruise flight is near the maximal efficiency, so we can consider it a constant.

Let the mass of the airplane without the accumulator be $m_{0}$ (according to the problem statement, $m_{0}$ is constant), $m$ is the mass of MAV, $g$ is the acceleration of gravity, $V$ is the flight velocity, $S$ is the wing area, $C_{L}$ is lift coefficient, $C_{D 0}$ is the drag coefficient at zero $C_{L}, W$ is consumed power, $\eta$ is the efficiency of the power plant, and $A$ is defined by

$$
A=\frac{1}{\pi e \lambda}
$$

where $\lambda$ is the aspect ratio and $e$ is a coefficient depending on the wing shape.

Then, for horizontal flight with constant velocity [4]

$$
m g=C_{L} \frac{\rho V^{2} S}{2}, W=\left(C_{D 0}+A C_{L}^{2}\right) \frac{\rho V^{3} S}{2 \eta}
$$

From this, it can be derived that

$$
W=\left(C_{D 0}+A\left(\frac{2 m g}{\rho V^{2} S}\right)^{2}\right) \frac{\rho V^{3} S}{2 \eta} .
$$

Let us find the velocity that corresponds to the minimal $W$.

It is well known [2] that for the small Reynolds numbers $\mathrm{Re}$ (which corresponds to the flight of MAVs)

$$
C_{D 0}=\frac{\text { const }}{\sqrt{\mathrm{Re}}}=\frac{B}{\sqrt{V}}=\frac{Z}{\sqrt{V b}}
$$

where $Z$ is a coefficient depending on wing form and kinematic viscosity of the air, $b$ is the characteristic wing chord, and $B=Z / \sqrt{b}$. equal to

Then the velocity corresponding to the minimal $W$ is

$$
V=\left(\frac{2 A}{5 B}\left(\frac{2 m g}{\rho S}\right)^{2}\right)^{\frac{2}{7}} \text {, or } V=\sqrt{\theta} m^{4 / 7},
$$

where $\theta=\left(\frac{2 A}{5 B}\left(\frac{2 g}{\rho S}\right)^{2}\right)^{4 / 7}$.
The mass of the accumulator $m_{\mathrm{AC}}$ is proportional to the $W$ and flight time $t$. Taking into account (1), (2) and (3), it can be found that

$$
m=m_{0}+k m^{\frac{10}{7}} t
$$

and

$$
t=\frac{m-m_{0}}{k m^{\frac{10}{7}}}
$$

where $k$ is a proportionality coefficient.

Varying $m$ as an independent variable, we will find that maximal flight time is realized for

$$
m=10 / 7 m_{\mathrm{AC}}, \text { or } m_{\mathrm{AC}}=7 / 3 m_{0},
$$

i.e., the mass of accumulator must be $70 \%$ of $m$.

\section{Optimization of power plant mass in the case of non-fixed airframe parameters for cruise flight}

It should be noted that the airplane mentioned above, even with an optimal accumulator, is not optimal as a whole: so it can fly with the increased mass, its airframe, electric drive, and control devices are "stronger" than necessary. Decreasing the mass and strength of these elements can improve the airplane and increase flight time. The problem of flight time optimization by the varying the masses of the accumulator, drive and airframe must therefore be analyzed.

It is well known that the masses of some elements of an airplane are proportional to the total airplane mass. Let the total mass of such elements be equal to $\alpha \cdot m$ ( $\alpha$ is a constant). The total mass of "constant" elements (i.e., those that are independent of $m$ and $W$ ) is equal to $m_{1}$. Assuming that the total mass of the electric drive and control devices is proportional to $W$ and using the previous results, we can find

$$
m=m_{1}+\alpha m+m^{\frac{10}{7}}(k t+\beta),
$$

or

$$
t=\frac{m(1-\alpha)-m_{1}}{k m^{\frac{10}{7}}}-\frac{\beta}{k},
$$

where $\beta$ is a proportionality constant.

Varying $m$ as independent variable, we determine that maximal flight time corresponds to

$$
m(1-\alpha)=\frac{10}{3} m_{1}
$$


or, in other words, the mass of the power plant must be $7 / 3$ of $m_{1}$.

\section{Dependence of flight time on airplane parameters}

Here the question of how the flight time depends on the aircraft parameters for task 2 must be investigated. Taking into account equation (3) and

$$
S=\frac{L^{2}}{\lambda}
$$

where $L$ is the wing span, one can find

$$
t=\frac{m_{A C}}{q W}=\sqrt[7]{\frac{5^{8} \cdot 2}{27}} \frac{\eta L^{\frac{6}{7}}(1-\alpha)^{\frac{10}{7}}}{q} \sqrt[7]{\frac{\rho^{3} \lambda^{2} \pi^{5} e^{5}}{B^{2} m_{1}^{3}}}-\frac{p}{q}
$$

where $p$ is "density of drive power" (i.e. drive mass divided by power).

Consider that

$$
b=\frac{L}{\lambda} \text { and } B=Z \sqrt{\frac{\lambda}{L}} .
$$

From this,

$$
t=\sqrt[7]{\frac{27}{16 \cdot 5^{5}}} \frac{\eta L(1-\alpha)^{\frac{10}{7}}}{2 q g} \sqrt[7]{\frac{\rho^{3} \lambda \pi^{5} e^{5}}{Z^{2} m_{1}^{3} g^{3}}}-\frac{p}{q}
$$

So, one can analyze the ways of flight time increasing according to formula (4). This expression shows that the best way of increasing $t$ is decreasing $q$. It is rather obvious that this way is simply the usage of accumulators with a higher amount of energy per unit mass.

The second way can be increasing the wing span, but in the case of the Micro vehicle the value of $L$ can only decrease. From this, we must keep in mind that the smaller the airplane is the shorter the flight time is.

In addition, rather efficient ways are increasing $\eta$ and $e$ and decreasing $\alpha$ and $p$. But these parameters can vary only slightly.

The effect of decreasing the "constant mass" $m_{1}$ is not as considerable as it is for the aforementioned parameters.

And lastly, increasing aspect ratio $\lambda$ does not yield a significant effect for this task.

\section{Optimization in the case of high Reynolds number}

This optimization procedure is the same as the previous case. The value of the optimal velocity can be obtained in the form

$$
V=\sqrt[4]{\frac{A}{3 C x_{0}}} \sqrt{\frac{2 m g}{\rho S}}, V=\sqrt{\gamma m}, \gamma=\sqrt{\frac{A}{3 C_{D 0}}} \frac{2 g}{\rho S},
$$

and the optimal accumulator mass is

$$
m=3 m_{0}, m_{\mathrm{AK}}=2 m_{0} .
$$

Maximum flight time is defined by the formula

$$
t=\frac{\eta L}{4 q \sqrt[4]{3}} \sqrt[4]{\frac{\lambda \pi^{3} e^{3}}{C x_{0}}} \sqrt{\frac{2 \rho}{3 g^{3}}} \sqrt{\frac{(1-\alpha)^{3}}{m_{1}}}-\frac{p}{q} .
$$

From this formula, one can see that the ways of increasing the value of flight time are the same as in the previous section.

\section{Behaviour of function $t$ near the maximum}

It is rather important to know the optimized function near the maximum. Figure shows the behaviour of the flight time of an airplane with a fixed airframe.

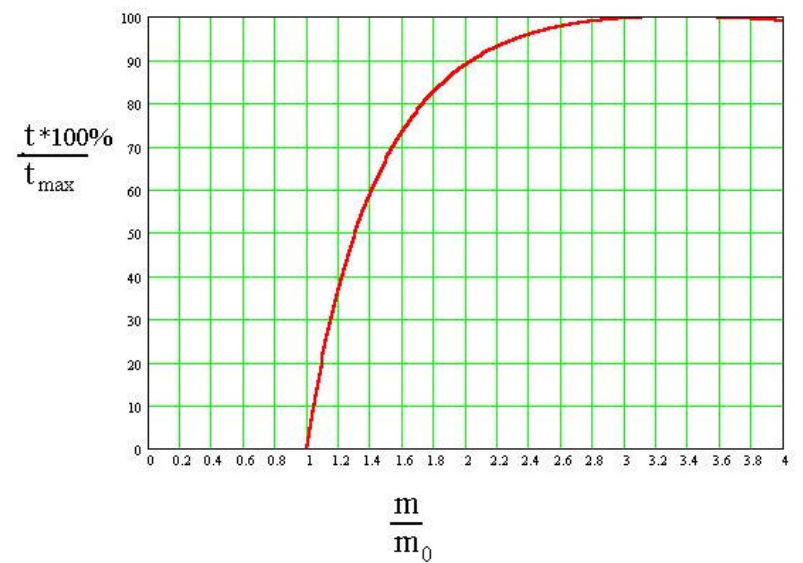

Fig. Flight time as function of total airplane mass for fixed airframe

From this figure, one can see that a decrease in accumulator mass on the value of $\mathrm{m}_{0}$ leads to a decrease in flight time of only $5 \%$. So, one can vary the accumulator mass significantly without a significant decrease in flight time.

\section{Comparison and validation of results}

Now one can compare the results obtained with the parameters of existing airplanes.

By Grasmeyer, JM. and Keennon, MT., mass breakdown for the first generation of the Black Widow configuration is as follows: power plant $-62 \%$, payload $-12 \%$, controls $-9 \%$, and structure $-17 \%$ of the total mass [1].

For the MAV Mirador these values are power plant $-75 \%$, structure $-11 \%$, and sensors, avionics and communication $-14 \%$ of total mass [2]. 
One can see that the masses of power plant for these MAVs are rather close to the value of $70 \%$ of the total mass (as in section 1). The difference in values can be explained, for example, by the fact that the variation in function near its maximum is rather small. Furthermore, the components of MAVs have a discrete set of masses, and for the majority of cases we cannot provide the equality of real masses and calculated masses.

\section{Conclusions}

1. In the case of low Reynolds numbers, the optimal accumulator mass for the maximization of flight time is $70 \%$ of total airplane mass, and for the case of high Reynolds numbers this value is $66 \%$ of total mass.

2. A decrease in accumulator mass of $30 \%$ from the optimal value leads to a decrease in flight time of about $5 \%$.

3. The value of maximal flight time linearly depends on wingspan, power plant efficiency, and the energy density of the accumulator. The aspect ratio of the wing affects flight time very slightly.

\section{Acknoledgements}

This work was carried out with the support of Russia's Presidential Grant for Young Scientists Support MK-5370.2006.8.

\section{References}

1. GRASMEYER, JM., KEENNON, MT. Development of the black widow micro air vehicle. In AIAA Paper, 2001, no. 2001-0127.

2. KEUTER, T., HERMANS, D., JÉRÔME, D. et al. Aerodynamic research on lifting surfaces for micro \& mini UAVs. Unmanned Air Vehicle Systems. Bristol, UK, April 08-10, 2002. In www.onera.fr/SEARCH/BASIS/public/web_fr/docum ent/DDD/315895.pdf

3. SEROKHVOSTOV, SV. Maximization of horizontal flight time for airplane with electrical power plant. Proceedings of the Fourth Seminar on "RECENT RESEARCH AND DESIGN PROGRESS IN AERONAUTICAL ENGINEERING AND ITS INFLUENCE ON EDUCATION", Part III. Warsaw: Institute of Aeronautics and Applied Mechanics, In Research bulleting, 2001, no 12, p. 131-133.

4. КОЛЕСНИКОВ, ГА. и др. Аэродинамика самолета. М.: Машиностроение, 1993. 